\title{
Canakinumab: can it untie the Gordian knot of cardiovascular disease in patients with familial Mediterranean fever?
}

\author{
Dimitrios Ioannis Patoulias
}

Second Propedeutic Department of Internal Medicine, General Hospital Hippokration, Aristotle University of Thessaloniki, Thessaloniki, Greece

Submitted: 27 May 2018

Accepted: 18 June 2018

Arch Med Sci Atheroscler Dis 2018; 3: e96-e98

DOI: https://doi.org/10.5114/amsad.2018.76866

Copyright $@ 2018$ Termedia \& Banach

Familial Mediterranean fever (FMF) is a monogenic autoinflammatory disease, characterized by inflammatory attacks of fever and polyserositis [1]. Missense mutations in the MEFV gene triggering abnormal activation of the pyrin inflammasome and the subsequent excessive production of interleukin-1 $\beta$ (IL-1 $\beta$ ) constitute the major underlying pathophysiological mechanism [2].

Patients with FMF are at high cardiovascular risk, which adds a significant burden to their quality of life, complicating at the same time their therapeutic management [3]. In their cross-sectional study with a prospective 3-year follow-up period, Yilmaz et al. observed that patients with FMF and secondary amyloidosis had increased risk of cardiovascular events, highly associated with elevated levels of asymmetric dimethyl arginine (ADMA), decreased flow-mediated dilatation (FMD) and an excessive inflammatory state [3].

Cakar et al. observed that patients with FMF during disease attacks had higher pulse wave velocity (PWV) and lower branchial and aortic augmentation indexes, both surrogate markers of arterial stiffness [4], when compared with asymptomatic patients and healthy controls [5]. Impaired arterial stiffness, as represented by PWV, correlated significantly with the main inflammatory markers, including serum C-reactive protein (CRP), erythrocyte sedimentation rate (ESR), white blood cells count (WBC), fibrinogen and neutrophil/lymphocyte ratio. In another cross-sectional study, Acay et al. observed that atherogenic index (triglycerides/ high-density lipoprotein-cholesterol) was significantly higher in patients with FMF compared to healthy controls [6]. The authors concluded that the atherogenic index may be an indicator of accelerated atherosclerosis in those patients, an observation that needs to be further elucidated in large-scale prospective studies.

A recently published study by Basar et al. shed light on the association between MEFV gene mutations and early coronary artery disease (CHD) [7]. More specifically, the researchers observed that MEFV gene mutations were significantly more frequent in patients with early CHD, when compared with those suffering from CHD in the presence of well-established risk factors or healthy controls. Interestingly, there were twice as many patients with early CHD and at least one MEFV gene mutation, compared with the two other study groups [7].

Puricel et al. conducted a retrospective clinical study, involving all patients admitted with acute coronary syndrome (ACS) aged less than

\author{
Corresponding author: \\ Dimitrios Ioannis Patoulias \\ Second Propedeutic \\ Department \\ of Internal Medicine \\ General Hospital \\ Hippokration \\ Aristotle University \\ of Thessaloniki \\ 3 B M. Alexandrou St \\ 57010 Thessaloniki, Greece \\ Phone: +30 6946900777 \\ E-mail: dipatoulias@gmail. \\ com
}


30 years in their department, during a 16-year period [8]. Acute coronary syndrome was of non-atherosclerotic (non-ATS) etiology in $37 \%$ of all patients, while FMF constituted a major underlying etiology in the latter group (7\%). The 5-year mortality and major adverse cardiovascular event (MACE) rates were $19 \%$ and $7 \%$ in the non-ATS and the ATS groups, respectively. Despite the presence of certain risk factors in both groups, mainly smoking and dyslipidemia, it is undoubtable that FMF is a major risk factor for cardiovascular disease, implicating both ATS and non-ATS mechanisms. On the other hand, there is contradictory evidence supporting that FMF is associated with lower incidence rates of certain cardiovascular and metabolic risk factors compared to healthy controls [9]. However, the aforementioned study conducted by Twig et al. lacks stratification of the results by the degree and severity of disease activity and genetic variants, due to the fact that the researchers included only military participants with FMF, who can be considered as healthier than other FMF patients [9].

Colchicine is considered as the first line treatment option in patients with FMF, with potential preventive action against cardiovascular disease, partially due to improvement in endothelial function $[10,11]$. However, it is ineffective or not tolerated in a significant proportion of those patients (5-10\%). In such cases, canakinumab, a monoclonal antibody against IL- $1 \beta$, offers an alternative treatment option in patients with colchicine-resistant disease [12].

It was recently reported in CLUSTER, a phase 3 clinical trial, that canakinumab led to a complete response at week 16 in $61 \%$ of all stratified FMF patients compared to $6 \%$ of those FMF patients assigned to placebo $(p<0.0001)$ [12]. In an explanatory analysis it was found that $71 \%$ of all colchicine-resistant FMF patients achieved a complete response with canakinumab ( $p<0.0001)$, while, after week 16 , an extended dosing interval of canakinumab bi-monthly led to disease control up to $46 \%$ in the same study group [12]. Despite the increased risk of infections associated with canakinumab administration, none was opportunistic, while all resolved without consequences.

Experimental data support that cholesterol crystals activate the NOD-like receptor pyrin domain containing protein inflammasome, which finally leads to the generation of $\mathrm{IL}-1 \beta$ and the triggering of a systemic inflammatory response [13]. The CANTOS trial, conducted by Ridker et al., investigated the potential role of canakinumab at three separate doses, 50, 150 or $300 \mathrm{mg}$, injected subcutaneously every three months, in cardiovascular disease [14]. The primary efficacy end point was nonfatal myocardial infarction, nonfatal stroke, or cardiovascular death. After a median follow-up period of 3.7 years, the researchers observed that hazard ratios (HR) for the primary endpoint for the three separate doses of canakinumab compared to placebo, were as follows: in the 50-mg group, 0.93 (95\% confidence interval $(\mathrm{Cl})$ : $0.80-1.07 ; p=0.30)$; in the $150-\mathrm{mg}$ group, 0.85 (95\% Cl: $0.74-0.98 ; p=0.021)$; and in the $300-$ mg group, 0.86 (95\% Cl: 0.75-0.99; $p=0.031)$. Regarding the secondary efficacy endpoint (primary endpoint plus hospitalization for unstable angina that required an urgent revascularization procedure), only canakinumab 150 mg led to a statistically significant result, in comparison with placebo $(\mathrm{HR}=0.83$; 95\% Cl: 0.73-0.95; $p=0.005)$. Canakinumab did not affect mortality rates among the different study groups [14].

However, a sub-analysis of the CANTOS trial showed that those patients who achieved hsCRP levels lower than $2 \mathrm{mg} / \mathrm{l}$ with canakinumab treatment had a $25 \%$ reduction in the incidence rate of major cardiovascular events (HRadj = 0.75; $95 \% \mathrm{Cl}: 0.66-0.85, p<0.0001)$, and $31 \%$ reduction in both cardiovascular (HRadj $=0.69 ; 95 \% \mathrm{Cl}$ : $0.56-0.85, p=0.0004)$ and all-cause mortality (HRadj $=0.69 ; 0.58-0.81, p<0.0001$ ), while those patients featuring greater inflammatory activity with hsCRP levels higher than $2 \mathrm{mg} / \mathrm{l}$ did not benefit from canakinumab treatment at a statistically significant level [15].

A closer look at the aforementioned data and a meticulous interpretation of the derived evidence leads to the conclusion that canakinumab might play a crucial role in terms of decrease in cardiovascular morbidity and mortality in FMF patients, constituting an attractive and promising treatment option. Another fact is that there is a distinct phenotype of patients, those with excessive inflammatory activity, who might benefit most from canakinumab administration. Randomized controlled trials with appropriate design and careful recruitment of FMF patients will elucidate whether canakinumab can untie the Gordian knot of cardiovascular disease in those patients.

\section{Conflict of interest}

The author declares no conflict of interest.

\section{References}

1. Ozen S, Batu ED, Demir S. Familial Mediterranean fever: recent developments in pathogenesis and new recommendations for management. Front Immunol 2017; 8: 253.

2. Ozçakar ZB, Yalçınkaya F. Vascular comorbidities in familial Mediterranean fever. Rheumatol Int 2011; 31: 1275-81.

3. Yilmaz MI, Demirkaya E, Acikel C, et al. Endothelial function in patients with familial Mediterranean fever- 
related amyloidosis and association with cardiovascular events. Rheumatology (Oxford) 2014; 53: 2002-8.

4. Mitchell GF, Hwang SJ, Vasan RS, et al. Arterial stiffness and cardiovascular events: the Framingham Heart Study. Circulation 2010; 121: 505-11.

5. Çakar M, Akhan M, Doğan T, et al. Investigation of the arterial stiffness and associated factors in patients with familial Mediterranean fever. Anatol J Cardiol 2017; 17: 132-8.

6. Acay A, Ulu MS, Ahsen A, et al. Atherogenic index as a predictor of atherosclerosis in subjects with familial Mediterranean fever. Medicina (Kaunas) 2014; 50: 329-33.

7. Basar N, Kisacik B, Ercan S, et al. Familial Mediterranean fever gene mutations as a risk factor for early coronary artery disease. Int J Rheum Dis 2017; 20: 2113-7.

8. Puricel S, Lehner C, Oberhänsli M, et al. Acute coronary syndrome in patients younger than 30 years: aetiologies, baseline characteristics and long-term clinical outcome. Swiss Med Wkly 2013; 143: w13816.

9. Twig G, Livneh A, Vivante A, et al. Cardiovascular and metabolic risk factors in inherited autoinflammation. J Clin Endocrinol Metab 2014; 99: E2123-8.

10. Erken E, Erken E. Cardiac disease in familial Mediterranean fever. Rheumatol Int 2018; 38: 51-8.

11. Pamuk BO, Sari I, Selcuk S, Gokce G, Kozaci DL. Evaluation of circulating endothelial biomarkers in familial Mediterranean fever. Rheumatol Int 2013; 33: 1967-72.

12. De Benedetti F, Gattorno M, Anton J, et al. Canakinum$a b$ for the treatment of autoinflammatory recurrent fever syndromes. N Engl J Med 2018; 378: 1908-19.

13. Duewell P, Kono H, Rayner KJ, et al. NLRP3 inflammasomes are required for atherogenesis and activated by cholesterol crystals. Nature 2010; 464: 1357-61.

14. Ridker PM, Everett BM, Thuren T, et al.; the CANTOS trial group. Antiinflammatory therapy with canakinumab for atherosclerotic disease. N Engl J Med 2017; 377: 1119-31.

15. Ridker PM, Everett BM, Thuren T, et al.; the CANTOS trial group. Relationship of $\mathrm{C}$-reactive protein reduction to cardiovascular event reduction following treatment with canakinumab: a secondary analysis from the CANTOS randomised controlled trial. Lancet 2018; 391: 319-28. 